\title{
EL DICCIONARIO COMO GÉNERO LITERARIO
}

Domingo García IBÁÑEZ Centro de Profesores y Recursos de Illescas (Toledo)

\section{Concepto de GÉnERo literario}

El concepto de Género literario ha sufrido numerosas modificaciones a lo largo de la Historia.

Vitor Manual Aguiar e Silva (Aguiar e Silva, V. M. 1984: 59), se pregunta por la existencia de los géneros literarios. Y se contesta a sí mismo argumentando que, dado que los valores literarios se supeditan al devenir histórico, lo más apropiado es adoptar una perspectiva diacrónica e ir analizando las soluciones que a este problema, al de los Géneros literarios, se han ido dando a lo largo del tiempo.

A continuación (p.p. 159/179 de la obra citada) hace un recorrido por las aportaciones realizadas a esta problemática, empezando en Platón y terminando en el estructuralismo.

Concluye con unas consideraciones acerca de la designación de «género»:

En el propio lenguaje crítico, el vocablo 'género' se refiere tanto a las grandes categorías de la lírica, de la narrativa y del drama, como a las diferentes especies englobadas en estas categorias - tragedia, comedia, novela, etc. Por eso proponen algunos críticos, a fin de evitar ambigüedades, la designación de formas naturales de la literatura pa- 
ra la lírica, la narrativa y la dramática, reservando la designación de géneros literarios para las especies históricas determinables dentro de aquellas formas naturales. Éstas representarían un concepto muy amplio, obtenido a partir de caracteres comunes extremadamente genéricos, mientras que los géneros constituirían un concepto más restringido, basado en elementos comunes, ya de la forma interna, ya de la forma externa, verificables en las obras abarcadas por cada una de las formas naturales (p. 179).'

Tradicionalmente la Teoría de la literatura ha clasificado las obras literarias en una serie de grupos; cada uno de ellos constituía un género literario y todas las obras pertenecientes a un determinado género poseían (y poseen) unas características comunes.

En nuestro artículo intentaremos demostrar que también los Diccionarios en general, y los de la Academia en particular, poseen unos rasgos comunes que los identifican como obras autónomas, como grupo característico, dentro del grupo universal de la Literatura.

Antes de proseguir quiero referirme, muy brevemente, al profesor $\mathrm{D}$. Francisco Abad Nebot, que ha sido el pionero en el tema que nos ocupa. Desde hace unos años el profesor D. Francisco Abad Nebot, de la Universidad Nacional de Educación a Distancia, imparte un curso de doctorado denominado precisamente "El Diccionario como Género Literario".

El doctor Abad (Abad Nebot, F, 2000: 355), siguiendo a Boris Tomachevski, esboza una teoría de los géneros literarios más dinámica en tanto que concibe el género literario como algo que se crea en el devenir de la historia mediante un proceso aglutinante.

Con cuatro notas sintetiza el profesor $\mathrm{Abad}^{2}$ los rasgos esenciales del género literario:

a) Las obras empíricas de una literatura se agrupan de modo natural según los procedimientos empleados en las mismas; tales procedimientos dan lugar a las características del género.

b) Esas características «son muy variadas, y pueden referirse a cualquier aspecto de la obra».

c) Si una obra tiene éxito, nace toda una literatura de imitaciones que es la que hace que exista el género.

Aguiar e Sil.va, Vitor Manuel., (1984): Teoría de la literatura. 6." Reimpresión. Madrid. Gredos.

2 Abad Nebot, Francisco, (2000), Cuestiones de lexicología y lexicografia, l." ed., Madrid, UNED. 
d) Los géneros viven y se desarrollan, y en cada obra particular del mismo se observan semejanzas o diferenciaciones respecto de los rasgos genéricos, esto es, la repetición o la variación de los procedimientos o rasgos constitutivos.

Concebido así el género literario, estamos en condiciones de afirmar que el Diccionario es un género literario en tanto en cuanto cada Diccionario es una obra que imita a otra anterior, que utiliza sus mismos procedimientos, que dispone de la misma forma sus artículos, los cuales a veces calca; ello no es obstáculo para que en otras ocasiones se produzcan innovaciones, variaciones en los redactados, recogida de nuevas acepciones y nuevas voces, a la vez que se desechan otras.

Hemos analizado esta permanencia de procedimientos y esas variaciones, esas «diferenciaciones respecto de los rasgos genéricos» a las que aludía el profesor Abad en su punto d) ya citado en una muestra de los Diccionarios de la Real Academia Española de 1984 y de 1992.

En concreto hemos analizado un corte de la letra «P», desde el inicio hasta la palabra «puchero». En total 127 páginas del Diccionario de 1984 (desde la página 991 a la 118 , ambas inclusive) y 136 páginas del Diccionario de 1992 (desde la página 1061 a la 1197, ambas inclusive).

Presentamos a continuación (sintetizados) algunos de los hallazgos de este análisis, hallazgos que vamos a agrupar en cuatro campos o capítulos:

- Fórmulas lexicográficas utilizadas.

- Contenidos de los artículos

- Diferencias diatópicas entre el léxico del D.R.A.E. de 1984 y el de 1992.

- Diferencias en la clasificación de las voces y expresiones entre los dos Diccionarios.

\section{FóRMULAS LEXICOGRÁfiCAS}

En ambos diccionarios se utilizan las siguientes fórmulas lexicográficas en la redacción de las definiciones:

- Acción o Acción y efecto.

- Aplícase (con la variante «Se aplica»).

- Calidad de

- Dícese (con la variante «Se dice»). 
- El que, La que, Lo que, seguidos de verbo.

- Especie de.

- Género o Cierto género de

- Perteneciente o relativo (con variantes).

- Que, seguido de verbo.

Podría pensarse quizá que la repetición de estas fórmulas lexicográficas obedece a una postura cómoda del lexicógrafo que se limita a «repetir» en el 92 lo que ya hiciera (él o algún colega) en el 84. Sin embargo, las alteraciones en el redactado de numerosos artículos (incluidos aquellos en los que aparecen estas fórmulas) y la utilización de estas fórmulas en voces que se incorporan por primera vez al Diccionario nos hace pensar que el empleo de estas estructuras obedece a lo que el profesor Abad señalaba en su punto c) al hablar del género literario: "Si una obra tiene éxito, nace toda una literatura de imitaciones que es la que hace que exista el género».

Analicemos, brevemente, cada una de estas fórmulas.

\subsection{Fórmula lexicográfica «Acción» o «Acción y efecto...»}

Esta formula es utilizada en muchas voces de ambos diccionarios que tienen un redactado idéntico en ambos (por ejemplo palabreo. m. Acción y efecto de hablar mucho y en vano).

No nos es posible (por la extensión que adquiriría el trabajo) enumerar todas las entradas que tienen idéntico redactado. Sí daremos su número: 243.

En las voces palmeo, preocupación, programación y propulsa aparecen, en el Diccionario del 92, acepciones nuevas que utilizan la fórmula lexicográfica que estamos utilizando.

Citamos, por último, aquellas voces que aparecen por primera vez en el Diccionario de la Academia en 1992 y que utilizan esta fórmula: palatalización, paletería, palmotada, plastificación, plastificado, da; posicionamiento, privatización y procrastinación.

\subsection{Fórmula lexicográfica «Aplícase» (y su variante «Se aplica»)}

\subsubsection{Fórmula «Aplícase»}

Ciento seis artículos utilizan esta fórmula, y redactan sus definiciones de forma prácticamente idéntica. Sirva como ejemplo la voz panóptico, ca. adj. 
Aplícase al edificio construido de modo que toda su parte interior se pueda ver desde un solo punto.

En cinco entradas (patrullero, ra; picoso, sa; positivo, va; practicante y providencial) aparecen nuevas acepciones que utilizan esta fórmula para sus definiciones.

Por último, las voces pivotante, premamá y prerromance aparecen recogidas por primera vez en el DRAE en la edición de 1992, y utilizan esta fórmula. Así, premamá. adj. Aplícase a la ropa o accesorios destinados a las mujeres embarazadas.

\subsubsection{Variante "Se aplica"}

Esta fórmula es utilizada en ambas ediciones en los artículos siguientes, los cuales están definidos de idéntica forma en una y otra edición: parejero, ra; pecaminoso, sa; pedicular; peliduro, ra; pietista; pintoresco, ca; pleistoceno, na; polifacético, ca; polivalente y protáceo, a.

\subsection{Fórmula lexicográfica «Calidad de»}

palatabilidad. f. Calidad de ser grato al paladar un alimento.

Así se define la voz "palatabilidad» en ambas ediciones ( 84 y 92) del DRAE. Esa misma circunstancia se da en otros 44 casos.

La entrada preterintencionalidad aparece recogida por primera vez en el DRAE en 1992, y es definida como: «Calidad de preterintencional. Puede considerarse circunstancia atenuante de la responsabilidad criminal».

\subsection{Fórmula lexicográfica «Dícese» (y su variante «Se dice»)}

Es una de las fórmulas más utilizadas, fórmula de la que disiente D. Manuel Seco porque (al igual que la de «Aplícase» que ya hemos visto) «quedan fuera de la ley de la sinonimia» ${ }^{3}$. Dice a este respecto D. Manuel (p. 24 de la obra citada):

Todas estas definiciones, encabezadas por 'dícese de' o 'aplícase a' -fómulas sumamente frecuentes a lo largo del Diccionario-.

${ }^{3}$ Seco, M. (1987), Estudios de lexicografía española. Madrid, Paraninfo, Ver p. 19 y ss. 
quedan fuera de la 'ley de la sinonimia'. Ni siquiera existe la identidad de categoría entre el definiente y el definido. La sustitución de misericordioso por su definición académica, en un texto como Es misericordioso con los que sufre, nos daría este otro texto, en modo alguno equivalente: (Es dícese del que se conduele y lastima con los trabajos ajenos, con los que sufren.

La explicación está, naturalmente, en que estas definiciones no son 'propias', sino 'impropias', es decir no son definiciones en metalengua de contenido, sino en metalengua de signo.

Sin embargo, el Diccionario de la R.A.E., que es al que se refiere D. Manuel Seco en la cita anterior, en su edición del año 1992 no solo mantiene esa fórmula en los artículos que «repite» del D.R.A.E. del año 1984 sino que la utiliza en las nuevas acepciones y en las nuevas voces que se incorporan por primera vez al Diccionario.

Hay que advertir que en la última edición del D.R.A.E. (la de 2001) se siguen manteniendo estas fórmulas aunque en otros casos se ha corregido la definición, separando lo que D. Manuel Seco denomina 'elementos habituales del contorno' (SECO y otros, 1999: XXII ${ }^{4}$ de la propia definición.

Veamos un par de ejemplo ilustrativos.

La acepción cuarta de la voz pacífico, ca, era definida en el D.R.A.E. de 1992 de la siguiente manera: «Dícese del sacrificio que ofrecían los gentiles por la paz y la salud; y por ext., del mismo sacrificio en la ley antigua de Moisés».

El D.R.A.E. del año 2001, define la acepción cuarta de esta misma voz de la siguiente manera: «Se dice del sacrificio que ofrecían los gentiles por la paz y la salud; y, por ext., del mismo sacrificio en la ley antigua de Moisés».

Como podemos apreciar la definición es prácticamente calcada (solamente se cambia «dícese» por «se dice», y se coloca entre comas la explicación «por extensión»).

Podríamos decir que, en este caso, se produce un continuismo en la fórmula definitoria.

Sin embargo, en otras ocasiones, se ha hecho caso de las advertencias de D. Manuel Seco, y se ha separado la definición estricta, de esos «elementos del contorno" a los que se refiere el ilustre académico.

Veámoslo con un ejemplo, el cual vamos a contrastar, además, con la definición que da D. Manuel Seco en su Diccionario.

4 Seco, Manuel y otros (1999): Diccionario del español actual, 1." Reimpresión. Madrid, Aguilar. 
Analicemos la voz palatal.

D. Manuel Seco y su equipo, en el Diccionario del español actual, definen la segunda acepción de esta voz de la siguiente manera: «(Fonét) [Articulación o sonido] que se realiza mediante la aproximación o contacto del dorso de la lengua y el paladar.»

Fiel a sus principios, D. Manuel Seco coloca entre corchetes la información que no afecta estrictamente a la definición, sino que forma parte del contomo de la palabra.

El D.R.A.E. de 1992, definía la segunda acepción de la voz que hemos elegido de la siguiente manera: «Fon. Dícese del sonido cuya articulación se forma en cualquier punto del paladar, y más propiamente de la vocal o consonante que se pronuncia aplicando o acercando el dorso de la lengua a la parte correspondiente del paladar duro, como la $i$ y la $\tilde{n}$.»

En la vigésima segunda edición del D.R.A.E. (año 2001)se define la segunda acepción de la voz que estamos analizando de la siguiente manera: «Fon. Dicho de una vocal o de una consonante: Que se articula aplicando o acercando el dorso de la lengua a la parte correspondiente al paladar duro; $p$. ej., la $i$ y la $\tilde{n}$. U.t.c.s.f.»

Tal como advierte la propia Academia (v. pp. XXVII y XLIII del Diccionario referido, edición en 1 tomo) «en los casos en que el Diccionario los presentaba fundidos, se ha separado el contorno de la definición, distinguiéndolo del contenido»; aunque podríamos añadir que no siempre se produce esta separación (como hemos comprobado en el lema pacífico). Con todo parece que la vigésima segunda edición del D.R.A.E. recoge algunas ideas sugeridas por D. Manuel Seco si bien este punto debería ser abordado con mayor rigor en estudios más completos.

Volviendo a la estructura que hemos mantenido a lo largo del artículo digamos que en 381 casos (empezando en el lema pacífico, ca y acabando en pubescente) se ha utilizado esta fórmula en ambas ediciones (la de 1984 y la de 1992, que son las que estamos analizando) con redactados prácticamente calcados.

En la edición de 1992 aparecen en el D.R.A.E. 34 acepciones nuevas, de voces que sí aparecían ya en la edición de 1984, definidas con esta fórmula y son 15 (desde pachanguero a presocrático, ca., las voces que son recogidas por primera vez en el D.R.A.E. y que están definidas con la fórmula que estamos analizando.

La fórmula «Se dice» es utilizada en 17 ocasiones en ambos Diccionarios, en artículos repetidos en ambas ediciones; es utilizada en la edición de 1992 en la definición de 3 acepciones nuevas. 


\subsection{Fórmula lexicográfica «(El, la, lo $)+$ que + verbo»}

Ilustramos la fórmula con la definición de la voz padronero, definición idéntica en ambos diccionarios: «m. ant. El que tiene derecho de patronato».

Esta fórmula es utilizada en 185 artículos que aparecen en ambas ediciones, $y$, en ocasiones, con definiciones muy parecidas $y$, a veces, idénticas.

Seis nuevas acepciones del D.R.A.E. de 1992 son definidas con esta fórmula, y la voz parental, que aparece recogida por primera vez en el D.R.A.E. de 1992 define su acepción segunda de esta manera: «Biol. Lo que se refiere a uno o a ambos progenitores.»

\subsection{Fórmula lexicográfica «Especie de»}

Artículos redactados con la misma fórmula en las dos ediciones (1984 y 1992): hay 81 artículos en el corte analizado.

En el D.R.A.E. de 1992 aparece recogida, por primera vez, la entrada parachispas, que es definida como «especie de pantalla metálica que se coloca en las bocas de las estufas o chimeneas de calefacción para impedir la salida de las chispas».

\subsection{Fórmula lexicográfica «Género o cierto género de»}

Las siguientes entradas están redactadas con la misma fórmula en ambas ediciones: palmilla, pantorrillera, pasador, ra; pasamano, picadillo, piñonata y piñonate.

\subsection{Fórmula lexicográfica «Perteneciente o relativo» 0 «Perteneciente»}

Esta fórmula presenta algunas variantes. Son las siguientes:

2.8.1. Artículos encabezados con «Perteneciente o relativo», tanto en el D.R.A.E. de 1984, como en el de 1992.

2.8.2. Artículos encabezados con «Perteneciente» tanto en el D.R.A.E. de 1984 como en el de 1992.

2.8.3 Artículos encabezados con «Perteneciente» en el D.R.A.E. de 1984, pero que en de 1992 añaden «o relativo». 
2.8.1. Artículos encabezados con "Perteneciente o relativo", tanto en el D.R.A.E. de 1984, como en el de 1992

En total hay 205. El primer lema en el que aparece esta fórmula es pajil («perteneciente o relativo a los pajes») y el último publicitario, ria.

2.8.2. Artículos encabezados con «Perteneciente» tanto en el D.R.A.E. de 1984 como en el de 1992

Hay un total de 81 artículos; el primero es pajuno, na («perteneciente a los pajes») y el último público, ca.

2.8.3. Artículos encabezados con «Perteneciente» en el D.R.A.E. de 1984, pero que en de 1992 añaden "o relativo".

Hay un total de 49 artículos que siguen esta pauta.

El primero es pacense, que en la edición de 1984 se registra con dos entradas para pasar, en la edición de 1992, a una sola entrada con dos acepciones distintas.

Hay otros artículos que emplean fórmulas muy próximas a las analizadas en este apartado: «Relativo o referente» o simplemente «Relativo». Aparecen (una o la otra) en 9 artículos; empiezan en paquidérmico, ca y terminan en protocolar ${ }^{2}$.

En el D.R.A.E. de 1992 aparecen 6 acepciones nuevas (en 6 artículos distintos) que utilizan la fórmula «Perteneciente o relativo».

Por último, para cerrar esta fórmula, digamos que aparecen recogidos por primera vez en el D.R.AE en 199216 artículos que utilizan alguna de las variantes señaladas anteriormente: El primero es paneuropeo y el último pucallpeño, ña.

\subsection{Fórmula lexicográfica «Que + verbo»}

Artículos redactados con la misma fórmula en las dos ediciones (1984 y 1992): hay 440 artículos redactados con esta fórmula, o con redactados similares, en ambos diccionarios. La primera palabra que aparece definida con esta fórmula es pacedero, ra. EI D.R.AE. de 1984 la define como «Que tiene hierba a propósito para pasto», mientras que en el de 1992 leemos «Que tiene hier- 
ba para pasto»; obsérvese la tendencia a simplificar la definición, otra de los rasgos distintivos del D.R.A.E. de 1992 con respecto al de 1984.

El último artículo que utiliza esta fórmula, dentro de la muestra analizada, es publicador, ra.

En el D.R.A.E de 1992 se recogen 22 acepciones nuevas que utilizan esta fórmula, y en este mismo Diccionario aparecen recogidas por primera vez 9 entradas que utilizan para su definición la fórmula que nos ocupa. Estas voces son: pasteurizado, da; pentavalente, perseguible, plateador, ra; preclusivo, preglaciar, preterintencional, propugnador y protuberante.

\subsection{Algunas conclusiones}

A. Muchos artículos del Diccionario de 1984 se repiten en la edición de 1992 sin sufrir variación alguna.

B. Algunas voces del diccionario de 1984, al ser definidas en el Diccionario de 1992 incluyen acepciones nuevas. Estas acepciones, a pesar de ser de nueva aparición, utilizan para la redacción de su definición las mismas fórmulas de encabezamiento que las que se utilizaban en la edición de 1984 en las acepciones ya definidas allí.

C. Algunas entradas que aparecen recogidas por primera vez en el D.R.A.E. de 1992,utilizan para la redacción de sus definiciones las mismas fórmulas de encabezamiento que utilizaban los artículos de 1984.

\section{CONTENido DE LOS ARTículos}

Existe una clara tendencia en el D.R.A.E. de 1992 a simplificar la redacción de los artículos y a utilizar un lenguaje más llano, menos artificioso que el que se emplea en el de 1984.

A veces es difícil apreciar esta simplificación pues muchos artículos tienen un redactado totalmente nuevo. En estos casos hemos recurrido a ver la extensión de uno y otro. Puede ocurrir, incluso, que la extensión sea igual (hemos recurrido incluso a contar el número de palabras que tiene una y otra redacción). Estos casos no son recogidos como simplificaciones, pero sí se aprecia ese gusto por el estilo más sencillo.

Veamos un ejemplo en el que la extensión del redactado es igual, pero en el que se puede apreciar fácilmente que en el D.R.A.E. DE 1992 el estilo es más sencillo, más llano, con menos giros. Escribimos, en primer lugar el artículo del D.R.A.E. de 1984 y, a continuación, debajo, el artículo del D.R.A.E. de 1992. 
profano, na. [...] || 2. Que es contra la reverencia debida a las cosas sagradas. II [...].

profano, na. [...] || 2. Que no demuestra el respeto debido a las cosas sagradas. II [...].

En este ejemplo las definiciones de las correspondientes acepciones constan del mismo número de palabras (diez), si bien la extensión, el espacio ocupado, puede ser ligeramente mayor en el 92 debido a que las palabras elegidas para redactar la definición son más largas; pero la definición está hecha con un lenguaje más sencillo, más directo.

Esta tendencia la hemos encontrado en otros 289 artículos del segmento analizado (desde pacción a puchero). Llama la atención la simplificación que se produce (apreciable a simple vista, sin necesidad de recuentos) en la entrada pájaro.

\subsection{Algunas conclusiones}

A. El Diccionario de 1992 tiende a reducir la extensión de las definiciones.

B. En el Diccionario de 1992 se observa, comparado con el Diccionario de 1984, una tendencia a utilizar un estilo más llano, menos artificioso, utilizando incluso estructuras empleadas en el registro coloquial.

4. DifERENCIAS DiatóPICAS ENTRE EL LÉXICO DEL D.R.A.E. DE 1984 Y EL DE 1992

Seguimos en este apartado a Eugenio Coserius ${ }^{5}$ (COSERIU, 1986 pp.303 y ss). Entendemos, por lo tanto, como diferencias diatópicas las diferencias geográficas que afectan a las significaciones.

El D.R.A.E. de 1992 incluye en bastantes artículos referencias a países hispanoamericanos que no aparecían en el 84. Se observa asimismo una tendencia a generalizar el uso de determinadas voces a América en general en lugar de restringir su uso a determinados países como hacía el Diccionario de 1984. En algunos casos, muy pocos, ocurre lo contrario, es decir, una voz que era usual en una zona geográfica más amplia, se ve reducida a un área geográfica menos extensa. Diferenciaremos unos casos y otros.

s COSERIU. E (1986): Lecciones de lingüística general, 1." Reimpresión, Madrid. Ed. Gredos. 
En el Preámbulo del Diccionario de 1992 se afirma, tras manifestar que se considera al castellano un «instrumento expresivo y conformador de una misma visión del mundo y de la vida» para todos los que lo utilizan, que

por eso ha solicitado insistentemente la Academia la cooperación de sus hermanas correspondientes y asociadas para dar mayor cabida en su DICCIONARIO a las peculiaridades léxicas y semánticas vigentes en cada país. Gracias a tal colaboración ha sido posible revisar y enriquecer en la presente edición el contingente americano y filipino.

Está claro que la Academia ha contado con información de la que no disponía en 1984 para proceder a efectuar estas modificaciones.

Vamos a ver unos ejemplos de cómo aparecen ampliadas las zonas geográficas de uso. Para mayor claridad sólo recogemos de los artículos las referencias geográficas. Colocamos un artículo debajo del otro; en primer lugar va el artículo del Diccionario de 1984.

pago $^{2}$. [...]. II 3. R. de la Plata.

pago $^{2}$. [...]. II 3. R. de la Plata y Perú.

paico. m. Chile.

paico. m. Amér. Meriod.

pailero, ra. m. y f. Ecuad. y Méj.

pailero, ra. m. y f. Ecuad., Méj. y Perú.

Esta ampliación de la zona geográfica de uso aparece en otros 43 artículos (desde paja hasta provocar).

\subsection{Acepciones nuevas}

Acepciones nuevas que afectan a zonas geográficas concretas. Se trata de entradas que ya recogía el Diccionario del 84 y en las que el del 92 añade la acepción a la que hacemos referencia, acepción que es específica del área que señalamos (sólo recogemos la referencia al área geográfica).

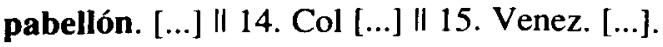

pacotilla. [...] $\|$ 2. Chile, Ecuad. y Guat.

pachocha. [...] $\|$ 2. Col. Cuba, Chile, Pan. y Perú.

pajonal. [...]. ॥ 2. Argent., Chile, Urug. y Venez.

pajuela. [...], I| 3. Bol., Col. y Chile. 
palenque. [...], || 4. Argent., Bol., Par., y Urug. palote. [...] II 3. Méj. [...].

\subsection{Entradas nuevas}

Entradas que aparecen por primera vez en el DRAE de 1992 y cuyo significado está restringido a un área geográfica concreta.

pacota. Argent.

pachanga. [...]. I| 2. pop. Méj.

pachiquil. N. de la Argent. [...] \| 2. N. Argent. [...]. || 3. N. Argent. padrillo. Argent., Chile, Par., Perú y Urug.

palanquear. Argent. y Urug.

palla. f. Argent. y Chile. [...]. II 2. Chile.

pallada. f. Amér. Merid.

\subsection{Artículos que restringen el área geográfica de uso}

pacho, cha. [...] $\|$ 2. Méj. y Nicar.

pacho, cha. [...] $\| 2$. Nicar.

pacayal. En el DRAE de 1992 desaparece la acepción 2, que se refería al uso de esta voz en Nicaragua.

paquetería $^{2}$. La acepción 2 , «Conjunto de prendas o adornos que una persona se pone para ir bien vestida», en el Diccionario del 84 aparece sin ninguna referencia geográfica, por lo que se entiende que es de uso común en todo el ámbito geográfico en el que se habla español. Sin embargo el Diccionario de 1992 especifica que esta acepción es de uso en Argentina, Paraguay y Uruguay.

Otros artículos que siguen esta tendencia son los siguientes: parición, parquear, pascana, pasote, payé, pelotear, pico $^{1}$, pilatuna, pileta, pinero, pisto, plato y playo, ya (en la acepción primera).

\subsection{Alguna conclusión}

El Diccionario de 1992 amplía las referencias americanas; además de ampliarlas trata con mayor rigor las diferencias diatópicas, ampliando, o restringiendo, las áreas geográficas de uso de las distintas voces. 


\section{DiferenCIAS EN LA ClasifiCACIÓN DE LAS VOCES Y DE LAS EXPRESIONES} Y LOCUCIONES ENTRE EL D.R.A.E. DE 1984 Y EL DE 1992

Se han producido, en la edición del Diccionario de 1992, algunos cambios en la clasificación de las palabras y de algunas expresiones y locuciones (muy pocas en el segmento analizado).

Algunos de esos cambios son poco significativos; se ha pasado de clasificar una voz como perteneciente al campo conceptual de la zoología, a considerarla perteneciente al campo de la biología, por ejemplo.

Vamos a analizar con detalle diez de estas palabras. Elegiremos cambios de distintos campos para las ejemplificaciones.

pacana.

Diccionario de 1984: f. Botánica.

Diccionario de 1992: $\mathrm{f}$.

En 1992 se generaliza su uso, no se clasifica en el campo conceptual de la Botánica.

pagar. Il pagarla, o pagarlas.

Diccionario de 1984: Expresión familiar.

Diccionario de 1992: Locución verbal familiar.

pagel.

Diccionario de 1984: $\mathrm{m}$. Zoología.

Diccionario de 1992: $\mathrm{m}$.

pájara.

Diccionario de 1984: Acepción 5 (mujer astuta): en sentido figurado.

Diccionario de 1992: Acepción 4 (mujer astuta): pasa a sentido real, no figurado.

\section{paladar.}

Diccionario de 1984: m. Anatomía.

Diccionario de 1992: m. Pasa a uso general, sin clasificación.

palmípedo, da.

Diccionario de 1984: adj. Zoología.

Diccionario de 1992: adj. Pasa a uso general, sin clasificación.

palo II de rosa II nefrítico.

Diccionario de 1984: Botánica.

Diccionario de 1992: Sin clasificación.

palotazo.

Diccionario de 1982: $\mathrm{m}$. Andalucía.

Diccionario de 1992: $\mathrm{m}$. Tauromaquia.

En 1992, por un lado se generaliza su uso, no se restringe a Andalucía (di- 
ferencia diatópico) y por otro se clasifica la palabra en el campo conceptual de la Tauromaquia.

palpitación. Acepción 3.

Diccionario de 1984: Fisiología.

Diccionario de 1992: Patología.

pancrático.

Diccionario de 1984: Zoología.

Diccionario de 1992: Anatomía.

Diferencias de este tipo aparecen en otros 87 artículos (continúa en pacaya y termina en pubes).

\subsection{Algunas conclusiones}

A. En la edición del Diccionario de 1992 se produce una reclasificación de bastantes entradas.

B. La mayoría de los cambios se producen debido a que la Academia estima (como resultado de sus investigaciones, suponemos) que, voces que en la edición de 1984 eran consideradas específicas de una ciencia o especialidad, han pasado, en el transcurso de estos años, a ser de uso común.

C. En otros casos el cambio en la clasificación se debe a una mayor precisión en la ubicación de la voz dentro de los campos científicos.

\section{OTROS ASPECTOS}

Antes de pasar a las conclusiones finales quisiera destacar otro aspecto de los Diccionarios que los acerca a las obras de otros géneros ya consolidados: su comportamiento en el mercado. Los diccionarios en general, y el Diccionario de la Real Academia en particular, siguen una pauta de comportamiento similar a la que siguen obras pertenecientes a otros géneros (novela, poesía, ensayo...). La última edición del Diccionario de la Real Academia ha acaparado, nada más aparecer, los primeros puestos en las listas de ventas. Así lo refleja El Cultural ${ }^{\circ}$ de la semana 7-13 de noviembre de 2001, en su página de "Libros más vendidos». El Diccionario de la lengua española de la R.A.E. aparece,

" El Cultural es un suplemento del diario El Mundo. Se entrega con el periódico de los miércoles. En este caso nos estamos refiriendo al suplemento «El Cultural» de la semana 7-13 de noviembre. pág. 10. 
dentro de la sección «No ficción», el número 1 en ventas, y es la segunda semana que ocupa ese lugar.

La estructura de las obras, y su comportamiento en el mundo editorial, nos hacen reiteramos en la defensa de que sean consideradas como un género específico, el género «Diccionario».

\section{Conclusiones generales}

7.1. El Diccionario de la Academia de 1984 y el de 1992 están elaborados mediante procedimientos similares.

7.2. A pesar de utilizar procedimientos similares, hay entre ambas ediciones ciertas diferencias que nos confirman que de una obra a otra se han producido variaciones.

7.3. Estas variaciones, a veces significativas, pueden afectar tanto al plano conceptual como al plano formal de los artículos.

7.4. Semejanzas. Entre las semejanzas entre ambas obras destacamos las siguientes:

\subsubsection{Repetición de artículos}

Algunos artículos aparecen en ambas obras no ya con la misma estructura sino también con idéntico contenido. Podríamos decir, en estos casos, que el artículo del Diccionario de 1992 es un calco del artículo del Diccionario de 1984.

7.4.2. Utilización de fórmulas lexicográficas para encabezar la redacción de los artículos

Hemos encontrado las siguientes fórmulas lexicográficas en el encabezamiento de la redacción de los artículos:

«Acción» o «Acción y efecto».

«Aplícase» (con la variante «Se aplica»).

«Calidad de».

«Dícese» (con la variante «Se dice»).

«El que», «La que», «Lo que», seguidos de verbo.

«Especie de».

«Género» o «Cierto género de». 
«Perteneciente o relativo» con variantes ya señaladas.

«Que, seguido de verbo».

7.5. Hay artículos del Diccionario de 1992 que son un calco de esos mismos artículos del Diccionario de 1984, y en los que se emplean estas fórmulas. Pero también aparecen acepciones o voces nuevas en el Diccionario de 1992 en las que se utilizan las fórmulas a las que nos referimos.

7.6. Lo dicho en el punto anterior demuestra que La Academia, en 1992, ha seguido utilizando estas fórmulas para las definiciones, a pesar de la crítica razonada de lexicógrafos de prestigio, como D. Manuel Seco, que defiende la tesis de que las definiciones encabezadas por "dícese de» o «aplícase a" son definiciones impropias por estar hechas en metalengua de signo en lugar de en metalengua de contenido.

7.7. Creemos, por lo tanto, que la utilización de estas fórmulas obedece a criterios prefijados, que no son producto del azar, y que están basadas en procesos imitativos.

7.8. Sin embargo, entre ambos Diccionarios se dan diferencias notorias, que señalaremos a continuación.

7.9. En el Diccionario de 1992 se utiliza un estilo más sencillo en la redacción de las definiciones, evitando las proposiciones subordinadas y utilizando estructuras y voces más próximas al registro coloquial.

7.10. El Diccionario de 1992 reduce la extensión de los artículos.

7.11. En la edición de 1992 se produce un mejor tratamiento del léxico americano.

\section{CONCLUSIÓN FINAL}

Los puntos enumerados anteriormente nos presentan dos obras que encajan en los rasgos esenciales de género literario expuestos al principio.

Consideramos que el Diccionario de 1992 presenta bastantes semejanzas, pero también numerosas diferencias con respecto al Diccionario de 1984, lo que le convierte en un continuador, pero con vida propia, de un género característico, que se distingue a su vez, de los ya existentes.

Por todo lo expuesto consideramos que se podría hablar con propiedad del género literario «Diccionario». 